\title{
Desarrollo de Pinus pseudostrobus bajo distintos ambientes de crecimiento en jardín común
}

\section{Development of Pinus pseudostrobus under different environments of growth in common garden}

\author{
Víctor Hugo Cambrón-Sandoval 1*, Humberto Suzán-Azpiri', Cuauhtémoc Sáenz-Romero C.? \\ y Naúm M. Sánchez-Vargas²
}

1 Facultad de Ciencias Naturales. Universidad Autónoma de Querétaro. Querétaro, México. hugo.cambron@gmail.com, hsuzan@uaq.mx.

* Autor de correspondencia

\author{
2 Instituto de Investigaciones Agropecuarias y Fores \\ tales. Universidad Michoacana de San Nicolás de \\ Hidalgo (IIAF-UMSNH). Morelia, Michoacán, México. \\ csaenzromero@gmail.com, \\ nsanchezv@yahoo.com.
}

\section{RESUMEN}

A partir de un análisis de componentes principales (ACP), se plantea la interpretación y clasificación de tres ambientes de competencia sobre el desarrollo de individuos de $P$. pseudostrobus integrando resultados previamente publicados dentro del mismo ensayo; las variables evaluadas fueron: el crecimiento (altura y diámetro a la base del tallo), producción de clorofila (cantidad de pigmentos de clorofila a y $b$ ), supervivencia, producción y distribución de biomasa total, raíz, rama, hoja y tallo, y arquitectura de crecimiento (largo de rama y altura de inserción de la primera rama) en familias de medios hermanos de $P$. pseudostrobus evaluados en un ensayo de jardín común a 10 meses de edad bajo tres ambientes de competencia. (I) baja densidad inter-familiar $(0.24 \times 0.12 \mathrm{~m})$, (II) alta densidad inter-familiar $(0.12 \times 0.06 \mathrm{~m})$ y $(\mathrm{III})$ alta densidad intra-familiar $(0.12 \times 0.06 \mathrm{~m})$. Se estimó que los tres componentes con mayor peso $(\mathrm{CP})$ explican el $82.9 \%$ de la variación total. El componente principal de mayor varianza se interpreta como crecimiento (CP1), agrupando a las familias por ambiente de competencia; al aumentar la densidad de plantación, posteriormente, las familias se agrupan por la capacidad de supervivencia y por la plasticidad fisiológica (CP2). Finalmente, la diferencia entre los ambientes la hace la plasticidad fenotípica de las familias como respuesta al ambiente de competencia (cambios en la arquitectura) (CP3). Los resultados evidenciaron un elevado nivel de plasticidad fenotípica de $P$. pseudostrobus y se hace hincapié en la importancia de la selección de ambientes y de genotipos acordes para aumentar la adaptación y productividad en los programas de mejora.

PALABRAS CLAVE: Análisis de componentes principales, competencia, estrategia de crecimiento, estrés.

\section{ABSTRACT}

From a principal component analysis (PCA), is is proposed the interpretation and classification of three main environments competition on the development of individuals of $P$. pseudostrobus integrating previous results within the same trial. The variables were: growth (height and base diameter), chlorophyll production (chlorophyll pigments total, $a, b$ and the ratio $a / b$ ), survival, production and distribution of dry weight biomass (total biomass of root, branch, leaf and stem) and growth architecture (length of branch insertion height and the lowest branch on the stem). All variables were measured in half-sibling families of $P$. pseudostrobus in a common garden test for 10 months-old seedlings in three competitive environments: (I) inter-family of low density $(0.24 \times 0.12 \mathrm{~m})$, (II) inter-family of high density $(0.12 \times 0.06 \mathrm{~m})$ and (III) intra-family with high density $(0.12 \times 0.06 \mathrm{~m})$. The first three components (PC) explained $82.9 \%$ of the total variation. The growth was the major component (PC1), grouping families for competitive environments as PC2 with families grouped by survivability and physiological plasticity, and finally, architectural structural changes as PC3. The results showed a high level of phenotypic plasticity of $P$. pseudostrobus, and emphasize the importance of the selection of environments and genotypes consistent to enhance adaptation and productivity improvement programs.

KEY WORDS: Principal component analysis, competition, growth strategy, stress. 


\section{INTRODUCCIÓN}

En México existen diversas especies del género Pinus como: P. patula, P. greggii, P. montezumae, etc., con potencial para ser utilizadas en plantaciones forestales comerciales en ambientes diversos (Perry, 1991). Una de esas especies es Pinus pseudostrobus Lindl, que fuera de su ambiente natural presenta gran variación en su rendimiento y desarrollo (Ferreira et al., 1972; Pires, 1987; Wright y Wessels, 1992). Diferentes estudios orientados a elevar la productividad de las plantaciones donde se ha manipulado la densidad de plantación, los nutrimentos o la cantidad de radiación solar disponible (Godin, 2000; Noland et al., 2001), han demostrado que las especies despliegan diferentes estrategias en su crecimiento, modificando los patrones en la distribución de los fotosintatos y las características morfométricas comunes (Barthélémy et al., 1995; Mutke et al., 2005), de tal manera que adoptan su propio modelo de arquitectura en el crecimiento. Muchos de los caracteres morfológicos de los individuos se desarrollan dependiendo de las características específicas de microambiente o de las restricciones ecológicas bajo las cuales crecen (Nishimura y Suzuki, 2001; Wright y Westoby, 2001) y, por lo tanto, se modifica su arquitectura fenotípica.

El ambiente de desarrollo de los individuos puede presentar variaciones o restricciones ecológicas (Wright y Westoby, 2001), de nutrientes (Kuuluvainen, 1992), de espaciamiento (Harms et al., 2000) o de tipo de competencia (Adams et al., 1973; Sánchez-Vargas y Vargas-Hernández, 2007; Cambrón-Sandoval et al., 2013a).

El análisis de componentes principales es una herramienta que permite entender cómo un conjunto de variables afectan las características del crecimiento (Jeffers, 1967; Liu y Keister, 1978; Real et al., 1989; Tardif et al., 2003), lo que permite identificar patrones de crecimiento asociados a ambientes específicos de desarrollo. El análisis de componentes principales es una técnica de reducción y de análisis de la estructura multivariada de los datos (Jolliffe, 1986) que transforma las variables originales en un pequeño conjunto de variables no correlacionadas (vectores propios o componentes principales) buscando que los primeros componentes expliquen la variabilidad de las variables originales (Jackson, 1993).

\section{OBjetivo}

El presente estudio integra los resultados de trabajos anteriores en los que se evaluaron variables de crecimiento (Cambrón-Sandoval et al., 2013a), fisiológicas (CambrónSandoval et al., 2011), de arquitectura y de asignación de biomasa (Cambrón-Sandoval et al., 2013b), con el objetivo de identificar patrones de crecimiento asociados a diferentes condiciones de competencia, identificando cambios en las características cuantitativas de crecimiento, fisiológicas y de arquitectura de la planta, que pueden ser importantes desde el punto de vista económico (altura, dap, densidad, etc.), en el inicio de programas de selección de genotipos capaces de adaptarse a condiciones ambientales futuras, ya sea por acciones de mejora y/o por presiones antropogénicas.

\section{MATERIALES Y MÉTODOS}

\section{Establecimiento del ensayo}

Para el establecimiento original del ensayo se utilizaron semillas de P. pseudostrobus de 13 árboles madre (familias), en lotes individuales, manteniendo su identidad por separado durante el estudio. Estas semillas fueron recolectadas en bosques naturales de la comunidad indígena de Nuevo San Juan Parangaricutiro, Michoacán, México, a una altitud entre $2200 \mathrm{~m}$ y $2900 \mathrm{~m}$; (19² $28^{\prime} \mathrm{LN}, 102^{\circ} 11^{\prime}$ LO a $\left.19^{\circ} 24^{\prime} \mathrm{LN}, 102^{\circ} 13^{\prime} \mathrm{LO}\right)$. Las semillas germinaron en condiciones de laboratorio, el proceso de germinación, consistió en un tratamiento pregerminativo $\left(8\right.$ días a $\left.5{ }^{\circ} \mathrm{C}\right)$, posteriormente las semillas se mantuvieron a temperatura ambiente en cajas de Petri, con riegos cada 48 h, durante 25 días y luego se trasplantaron a contenedores rígidos cooperblocks de $220 \mathrm{~cm}^{3}$ de capacidad (dimensiones: 119 $\mathrm{mm}$ de alto, $55 \mathrm{~mm}$ de diámetro superior e inferior) con sustrato compuesto por musgo sphagnum, perlita expandida y vermiculita en proporción de 2:1:1 en volumen, en donde crecieron por tres meses. Después, las plántulas fueron establecidas en un ensayo de jardín común en 
Morelia, Michoacán, México (ubicado a los $19^{\circ} 46^{\prime} 7,4$ ” N y $101^{\circ} 8$ ' 56,6” O).

El ensayo se realizó bajo un diseño de jardín común, ya que se considera como una etapa intermedia entre vivero (en donde se tiene una limitante por el espacio del envase) y el campo (en donde los efectos de micrositio no son controlados). Para eliminar el efecto de la variación inicial, tanto en altura como en diámetro a la base dentro del ensayo, se realizó una medición cero, evaluando las plantas en altura y diámetro a la base al momento de integrarse al ensayo. Posteriormente, esta medición se utilizó como una covariable al momento del análisis. El cajón de crecimiento consistió en un cajón rectangular de $15 \mathrm{~m}$ de largo x 1,2 m de ancho x 0,6 m de alto, llenado con capas de $20 \mathrm{~cm}$ de espuma volcánica gruesa, $5 \mathrm{~cm}$ de tezontle, $10 \mathrm{~cm}$ de tierra de encinar y $20 \mathrm{~cm}$ de turba de musgo sphagnum, perlita expandida y vermiculita en proporción de 2:1:1 en volumen. Contó con un sistema de riego automatizado a través de nebulizadores ubicados cada 1,15 m de distancia, a una altura de 1,90 m, con una capacidad de riego de $125 \mathrm{~cm}^{3} / \mathrm{min}$. Con periodos de riego de 20 minutos cada tercer día (sólo en época de estiaje se realizaron riegos diarios).

El ensayo original se estableció bajo un diseño de parcelas divididas, con cuatro repeticiones y cuatro individuos como unidad experimental; las parcelas grandes fueron las condiciones de competencia y las chicas, las familias. Se probaron tres condiciones: I) baja competencia inter-familiar, con familias mezcladas, plantadas a un espaciamiento de 0,25 $\mathrm{m} \times 0,12 \mathrm{~m}$; este ambiente actuó como referencia de no competencia ya que permitió el libre desarrollo de las plantas en las primeras etapas de crecimiento; II) alta competencia inter-familiar, con familias mezcladas plantadas a 0,12 $\mathrm{m} \times 0,06 \mathrm{~m}, \mathrm{y}$ III) alta competencia intra-familiar, con familias plantadas en subparcelas unifamiliares a 0,12 $\mathrm{m} \times 0,06$ $\mathrm{m}$. Se establecieron fajas de protección en los perímetros de cada ambiente para evitar el efecto de borde y dentro del ambiente de competencia intra-familiar (III) se establecieron sub-parcelas de 16 plantas, utilizando sólo las cuatro centrales para el análisis estadístico, ya que se encontraban rodeadas por individuos de la misma familia (Fig. 1).
Las condiciones I y II permitieron evaluar el efecto del nivel de competencia asociada a la densidad de plantación y las condiciones II y III el efecto del tipo de interacción genotípica (inter-familiar $v s$. intra-familiar).

\section{Variables evaluadas}

El presente trabajo integra resultados de estudios previos, bajo el mismo diseño de jardín común y con las mismas familias evaluadas, identificando posibles patrones de desarrollo como respuesta al ambiente de competencia. Las variables evaluadas en dichos estudios fueron: variables de crecimiento (altura y diámetro a la base del tallo) (Cambrón-Sandoval et al., 2013a), evaluadas cada mes durante 10 meses; cantidad de pigmentos de clorofila expresadas en clorofila total, clorofila $a$, clorofila $b$ y la relación de clorofilas $a / b$ (partes de pigmentos de clorofila a por clorofila b) (Cambrón-Sandoval et al., 2011) a 10 meses de establecido el ensayo; y, finalmente, la supervivencia, producción y distribución de biomasa (biomasa total, de raíz, de tallo, de hoja y de rama), se cosecharon los individuos a 11 meses de establecido el ensayo (Cambrón-Sandoval et al., 2013b).

\section{Análisis de datos}

Previo al análisis de los resultados se evaluó la normalidad de los datos a partir del procedimiento UNIVARIATE (normal test) de SAS (SAS, 1999). Para la variable supervivencia, en los diferentes ambientes de competencia, se realizó la transformación de los datos a partir de arcoseno para cumplir el supuesto de continuidad de los datos. Así, para evaluar el efecto del ambiente de competencia sobre la supervivencia, la producción de biomasa y arquitectura de la planta, se hizo un análisis de varianza (ANOVA) explorando las posibles diferencias entre las familias, con el procedimiento MIXED de SAS (1999), mediante el siguiente modelo, donde el factor $C$ se consideró efecto fijo:

$$
Y_{i j k l}=\mu+B_{i}+C_{j}+B C_{i j}+F_{k}+C F_{j k}+B C F_{i j k}+e_{i j k l}
$$

Donde: $Y_{i j k l}$ es el valor observado del $l$-ésimo individuo en la $k$-ésima familia, en el $j$-ésimo ambiente de competencia 


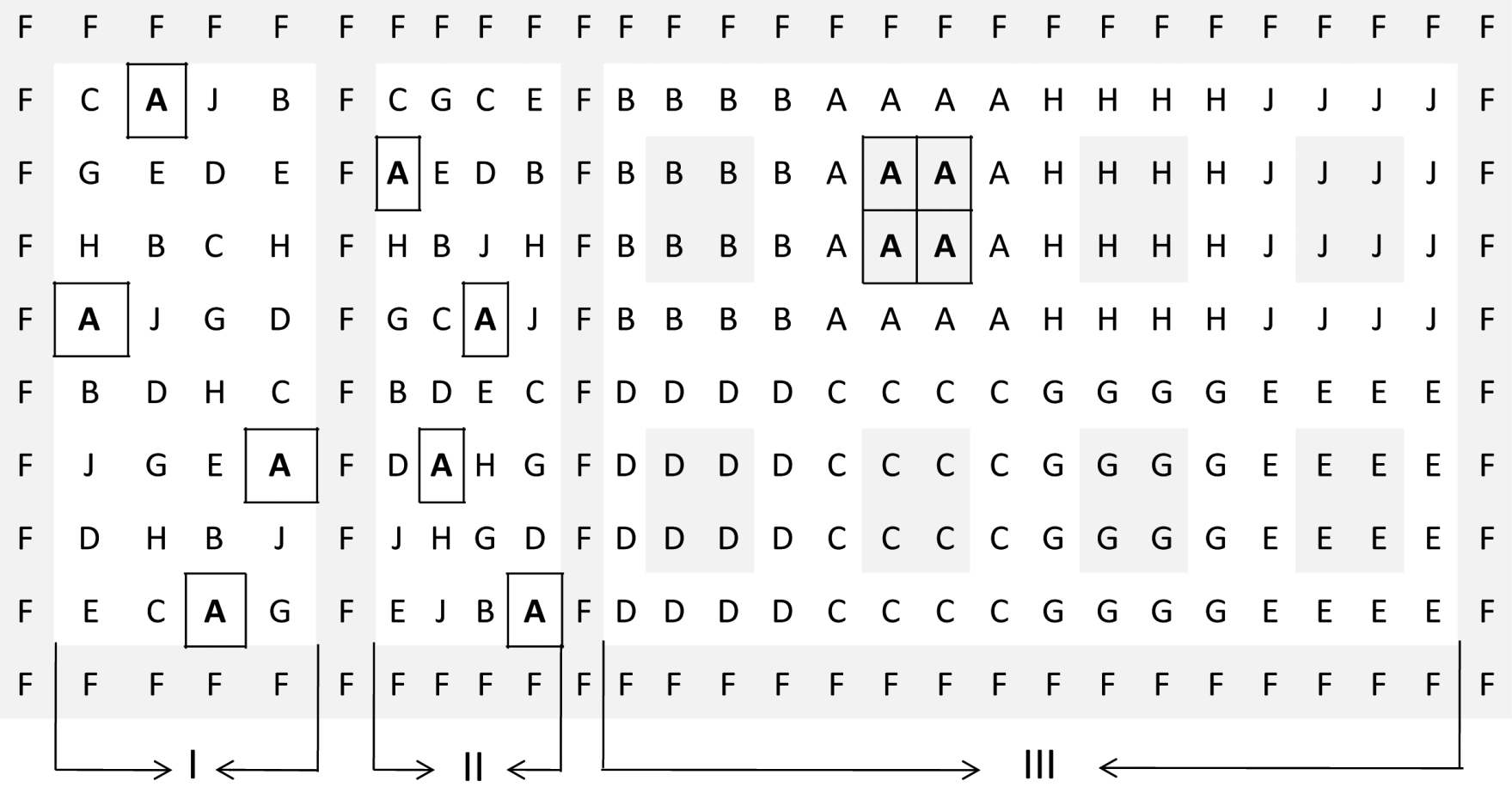

Figura 1. Ensayo de jardín común, bajo un diseño de parcelas divididas de Pinus pseudostrobus Lindl. (ambiente I, ambiente II y ambiente III).

en el $i$-ésimo bloque; $\mu$ es el valor promedio de la población; $B_{i}$ es el efecto del $i$-ésimo bloque; $C_{j}$ es el efecto fijo del $j$-ésimo ambiente de competencia; $B C_{i j}$ es el efecto de la interacción del $i$-ésimo bloque con el $j$-ésimo ambiente de competencia (error de la parcela grande); $F_{k}$ es el efecto aleatorio de la $k$-ésima familia $\left[\left(F_{k}\right)=0 ; \operatorname{Var}\left(F_{k}\right)={ }_{\mathrm{f}}^{2}\right] ; C F_{j k}$ es el efecto de la interacción del $j$-ésimo ambiente de competencia con la $k$-ésima familia $\left[\left(C F_{j k}\right)=0\right.$; $\operatorname{Var}\left(C F_{j k}\right)=$ $\left.\sigma^{2}{ }_{c f}\right] ; B C F_{i j k}$ es la interacción del $i$-ésimo bloque con la $k$-ésima familia en el $j$-ésimo ambiente de competencia $\left[\left(\mathrm{BCF}_{i j k}\right)=0 ; \operatorname{Var}\left(\mathrm{BCF}_{i j k}\right)=\sigma_{\text {bcf }}^{2}\right]$ (error de la parcela chica); y $e_{i j k l}$ es el error dentro de parcela $\left[\left(e_{i j k l}\right)=0\right.$; $\left.\operatorname{Var}\left(e_{i j k l}\right)=\sigma^{2}{ }_{\mathrm{e}}\right]$.

El grado de asociación del ambiente de competencia con las variables que determinan el desarrollo de los individuos, se estimó a partir de un análisis de componentes principales (CP), a través del procedimiento PRINCOMP STD de SAS (1999), identificando patrones de crecimiento como efecto del ambiente de competencia en donde se desarrollaron los individuos, se seleccionó el CP de tipo R (en función de los coeficientes de correlación), ya que éste permite trabajar con variables que presentan magnitud y unidades de medidas diferentes (Pla, 1986). Previo al análisis final, se eliminaron variables como: altura, clorofila total, clorofila $a$, clorofila $b$ y biomasa total, por presentar elevados valores de correlación $\left(r^{2}>0,85\right)$, esto bajo el criterio de Kaiser (1958) y Jackson (1993). Así, las variables candidatas al análisis de componentes principales fueron: diámetro a la base del tallo, largo de rama, altura de inserción de la rama más baja en el tallo, relación de pigmentos de clorofila $a / b$, biomasa de raíz, de hoja, tallo y rama.

\section{RESULTADOS Y DISCUSIÓN}

\section{Efecto del ambiente de competencia}

En general, los resultados de las variables evaluadas evidenciaron que el factor de mayor impacto en el ensayo fue 
la reducción del espacio, con los valores más elevados en todas las variables dentro del ambiente de libre crecimiento (I) (Tabla 1). Lo anterior excepto en la clorofila $b$, donde, el ambiente de competencia III expresó el mayor incremento (Tabla 1), además de la altura de inserción de la rama más baja en el tallo, en donde las condiciones de alta densidad (II y III) insertaron las ramas iniciales cerca de la parte apical del tallo, estimando diferencias significativas $(P \leq 0,05)$ entre condiciones de competencia.

\section{Análisis de componentes principales}

A partir del análisis integrador de componentes principales, se transformaron las variables originales en un pequeño conjunto de variables, donde los tres primeros componentes principales en el análisis general $(\mathrm{CP} 1=$ Crecimiento, $\mathrm{CP} 2=$ Supervivencia y fisiología y CP3 $=$ Arquitec - tura) explican $82,9 \%$ del total de la variación observada. Los vectores característicos de las variables consideradas como de "crecimiento" (diámetro a la base del tallo, biomasa de tallo, hoja y rama) contribuyeron de manera muy semejante al primer componente principal (CP1) (Tabla 2). Para el caso del segundo de los componentes (CP2), las variables que determinan parte de la arquitectura en el crecimiento (altura de inserción de la primera rama en el tallo), además de la supervivencia y el ajuste fisiológico a partir del cociente entre la cantidad de pigmentos de clorofilas $a$ y $b$ (partes de pigmentos de clorofila $a$ por clorofila $b$ ), aportan los valores más elevados, siendo la supervivencia la de mayor aportación, por lo cual el componente se denominó “supervivencia”. Finalmente para el tercer componente (CP3) el vector de mayor aporte fue el largo de rama, variable que determina la arquitectura

TABla 1. Promedios por ambiente de competencia en variables de crecimiento, fisiológicas, supervivencia, biomasa en peso seco y arquitectura de plantas de Pinus pseudostrobus de 12 meses de edad.

\begin{tabular}{|c|c|c|c|c|c|c|}
\hline \multirow{2}{*}{ Variable } & \multicolumn{4}{|c|}{ Ambiente de competencia } & \multirow{2}{*}{\multicolumn{2}{|c|}{ III }} \\
\hline & I & & II & & & \\
\hline Altura (cm) & 61,40 & a & 48,40 & $\mathrm{~b}$ & 47,60 & $\mathrm{~b}$ \\
\hline Diámetro a la base del tallo (cm) & 11,48 & a & 8,08 & b & 7,54 & $\mathrm{~b}$ \\
\hline Clorofila total (g m-2) & 0,5927 & a & 0,5274 & $\mathrm{~b}$ & 0,5262 & $\mathrm{~b}$ \\
\hline Clorofila a $\left(\mathrm{g} \mathrm{m}^{-2}\right)$ & 0,4956 & a & 0,4267 & $\mathrm{~b}$ & 0,3758 & $\mathrm{~b}$ \\
\hline Clorofila b ( $\left.\mathrm{g} \mathrm{m}^{-2}\right)$ & 0,0951 & b & 0,0907 & b & 0,1003 & a \\
\hline Relación de clorofilas a/b & 5,20 & a & 4,10 & b & 3,40 & c \\
\hline Supervivencia (\%) & 97,24 & a & 75,18 & $\mathrm{~b}$ & 72,88 & $\mathrm{~b}$ \\
\hline Biomasa total (و) & 91,24 & a & 44,15 & $\mathrm{~b}$ & 43,63 & b \\
\hline Biomasa de raíz (و) & 22,04 & a & 15,28 & b & 15,98 & $\mathrm{~b}$ \\
\hline Biomasa de hojas (و) & 40,89 & a & 16,06 & b & 16,28 & $\mathrm{~b}$ \\
\hline Biomasa de tallo (g) & 23,60 & a & 11,73 & b & 9,19 & $\mathrm{~b}$ \\
\hline Biomasa de ramas (g) & 4,71 & a & 1,07 & $\mathrm{~b}$ & 2,18 & $\mathrm{~b}$ \\
\hline Longitud de rama (cm) & 15,77 & a & 7,75 & c & 9,07 & b \\
\hline Altura de inserción de rama (cm) & 12,68 & c & 36,35 & a & 25,31 & $\mathrm{~b}$ \\
\hline
\end{tabular}

I = Baja densidad inter-familiar; II= Alta densidad inter-familiar; III= Alta densidad intra-familiar. †Valores promedio en una misma fila seguidos de letras diferentes representan diferencias significativas (Tukey, $P<0,05$ ) entre ambientes de competencia. 
del crecimiento, denominando el tercer componente como "arquitectura" (Tabla 2).

El componente principal uno (CP1), lo integran principalmente variables que explican el crecimiento en los individuos. Se evidenció una agrupación de los individuos con los mayores promedios de crecimiento, correspondiendo en su totalidad a familias que se desarrollaron dentro del ambiente de libre competencia (I) (Fig. 1). En contraparte, en las condiciones de mayor densidad de plantación (II y III), los individuos presentaron desarrollos menores, reflejando diferencias significativas $(\mathrm{P} \leq 0,05)$ entre densidades de plantación (I $v s$ II y I $v s$ III), pero no entre condiciones de competencia inter e intra-familiar (II vs III) (Cambrón-Sandoval et al., 2013a).

Para el componente principal dos (CP2), los vectores que determinan el peso son: la supervivencia, valor que se reduce entre densidades de plantación (I $v s$ II y I $v s$ III) (Tabla 1), y la altura de inserción de la rama más baja en el tallo, observando diferentes comportamientos entre condiciones de competencia, modificando la arquitectura de los individuos, dentro de las condiciones de mayor densidad (II y III), donde la rama más baja en el tallo se presentó cerca de la parte apical del árbol. En contra parte, dentro del ambiente de baja densidad (I), la rama se insertó cerca de la base del tallo (Tabla 1). Finalmente, la cantidad de pigmentos de clorofila $a$ por pigmentos de clorofila $b$ (relación de clorofilas $a / b$ ), expresaron diferencias significativas entre condiciones de competencia $(P \leq 0,05)$, evidenciando una agrupación de las familias por ambiente de competencia (Fig. 2), especialmente, dentro del ambiente de alta densidad intra-familiar (III), condición en donde los individuos evidenciaron mayor nivel de estrés fisiológico, en relación con las condiciones de competencia interfamiliares (I y II) (Cambrón-Sandoval et al., 2011).

En Pinus pinea L. se han observado cambios fisiológicos y su repercusión en el crecimiento de los individuos como respuesta a modificaciones del ambiente de desarrollo (Sánchez-Gómez et al., 2011), en donde, el análisis de componentes principales agrupó las familias de acuerdo con la cantidad de clorofila y con el incremento del creci-

TABLA 2. Autovalores y vectores característicos del análisis de componentes principales de Pinus pseudostrobus bajo distintos ambientes de competencia y aportaciones a la variación total por componente (Los valores en las casillas de las variables son los coeficientes del eigenvector).

\begin{tabular}{lccc}
\hline Variable & $C P 1$ & $C P 2$ & $C P 3$ \\
\hline Autovalor & $\mathbf{5 , 6 6 9}$ & 1,024 & 0,772 \\
\hline Aportación a la varianza total & $\mathbf{6 3 , 0 \%}$ & $11,4 \%$ & $8,5 \%$ \\
\hline Diámetro a la base & $\mathbf{0 , 3 9 4 4}$ & 0,1926 & $-0,0218$ \\
\hline Biomasa de raíz & 0,3438 & $-0,1485$ & $-0,1835$ \\
\hline Biomasa de hoja & $\mathbf{0 , 4 0 3 3}$ & $-0,0188$ & $-0,1083$ \\
\hline Biomasa de tallo & 0,3854 & 0,1017 & $-0,1426$ \\
\hline Biomasa de rama & 0,3384 & $-0,3046$ & 0,1306 \\
\hline Largo de rama & 0,2410 & $-0,3402$ & 0,7623 \\
\hline Altura de inserción de rama & $-0,2737$ & 0,5057 & 0,2996 \\
\hline Relación de clorofilas $a / b$ & 0,3283 & 0,4051 & $-0,2454$ \\
\hline Supervivencia & 0,2440 & 0,5492 & 0,4305 \\
\hline
\end{tabular}

$\mathrm{CPl}=$ Componente principal $1, \mathrm{CP} 2=$ Componente principal 2 y $\mathrm{CP} 3=$ Componente principal 3 Nota: Mayores aportaciones dentro del componente principal se resaltan en negritas. 


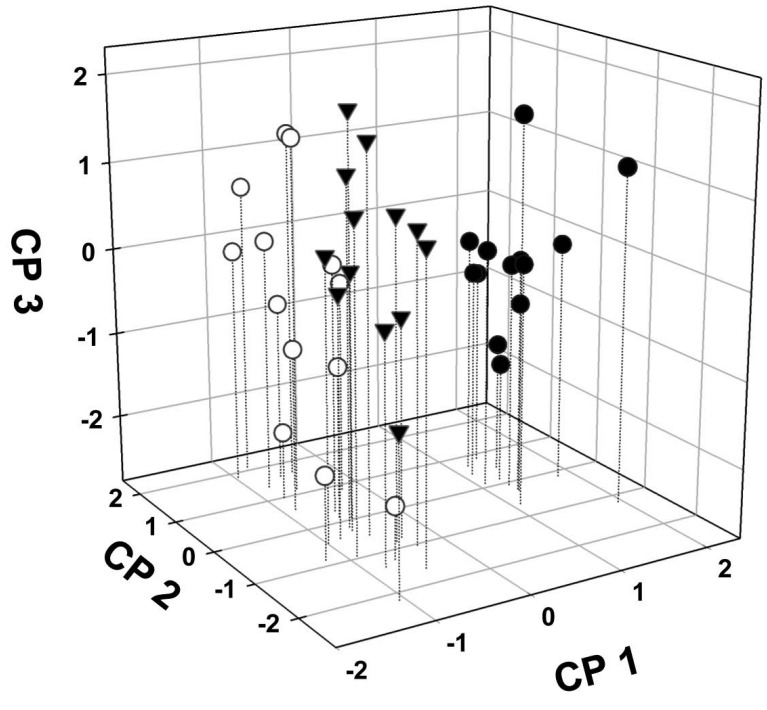

FIGURA 2. Componentes principales: (CP1) componente de crecimiento, (CP2) componente de supervivencia y (CP3) componente de arquitectura, en $P$. pseudostrobus, bajo distintas condiciones de competencia. Donde: $\bullet$ = Baja densidad inter-familiar, $\bigcirc=$ Alta densidad inter-familiar y $\boldsymbol{\nabla}=$ Alta densidad intra-familiar.

miento explicando 55,0\% de la variación total bajo diferentes índices de aridez, dentro de una cámara de crecimiento. Cuando los individuos crecían con un nivel de estrés superior (mayor índice de aridez), presentaban menor cantidad de pigmentos de clorofila por unidad de superficie $(-32,5 \%)$ y el crecimiento era inferior $(-41,8 \%)$ en relación a individuos que se desarrollan bajo menor estrés (índice de aridez menor). Un efecto similar se estimó con Pinus pinaster Ait., en el cual los individuos sometidos a un nivel de sombreo mayor (146 arboles ha ${ }^{-1}$ ) (como efecto de una alta densidad de población) presentan un desarrollo en altura inferior y menor cantidad de pigmentos de clorofila por unidad de superficie, en comparación con individuos establecidos en ambientes de baja densidad de población (56 arboles ha-1), agrupándose dentro del análisis, en individuos tolerantes e intolerantes a la sombra y con diferencias en el crecimiento (Rodríguez-García et al., 2011). Estos resultados son altamente similares a los obtenidos en el presente estudio, en donde se obtuvieron patrones de agrupación de las familias de acuerdo con el ambiente de competencia en donde se desarrollaron. Por lo tanto, la relación de la cantidad de pigmentos de clorofilas $a / b$ fueron modificados y se identificaron dos grupos en el análisis de componentes principales, explicando una escala de nivel de estrés de los individuos dentro del ensayo. El ambiente I representaría el menor nivel de estrés, el ambiente II el nivel intermedio y el ambiente de competencia III el nivel máximo de estrés. Se considera el máximo nivel de estrés por presentar características de espacio reducido para el crecimiento y estar rodeado por individuos genéticamente similares (similitud de respuesta al ambiente).

Dentro del componente principal tres (CP3), el largo de rama fue el vector de mayor aporte (Tabla 2). Se observaron ramas de mayor longitud dentro del ambiente de baja densidad inter-familiar (I), con diferencias significativas $(\mathrm{P}=0,05)$ tanto entre densidades de plantación (I vs. II y I vs. III) (Tabla 1) como entre condiciones de plantación (II vs. III), con los menores promedios de longitud de ramas dentro del ambiente de alta densidad inter-familiar (II). Sin embargo, dentro del análisis de componentes principales no se reflejó una agrupación por ambiente de competencia (Fig. 2).

En estudios en donde se analiza la conformación de la arquitectura del crecimiento como respuesta al medio ambiente, se ha observado que la densidad de plantación puede modificar la arquitectura en el desarrollo de los individuos. Harms et al. (1994) estimó, en Pinus taeda L. bajo condiciones de campo, que individuos que crecieron en condiciones de mayor densidad (1,8 $\mathrm{m} \times 1,8 \mathrm{~m})$, presentaron un menor crecimiento y el largo promedio de sus ramas fue inferior, en comparación con individuos que crecieron en ambientes con una menor densidad de plantación (3,7 m × 3,7 m). De la misma forma Mutke et al. (2005), en estudios con injertos de Pinus pinea y Pinus taeda, observaron que la conformación de la copa (largo de ramas y altura de copa) es modificada en individuos que son sometidos a un estrés excesivo, expresado por condiciones de suelos pobres y alta densidad en la población; indicando que los injertos que crecen en ambientes de alta densidad de plantación y en suelos carentes de 
nutrientes, el promedio del largo de ramas y la altura de la copa se reduce, en comparación con los injertos que crecen en ambientes de baja densidad de plantación y en suelos con calidad de nutrimentos aceptable. Estos resultados, reflejan similitud al presente estudio en la conformación de la arquitectura del crecimiento como efecto del ambiente de competencia, ya que las familias de $P$. pseudostrobus evaluadas presentan diferencias significativas en el largo promedio de la rama $(\mathrm{P} \leq 0,05)$ entre todas las condiciones de competencia.

\section{Estrategias de las familias dentro de los diversos ambientes de competencia}

Dentro de cada ambiente de competencia específico, el porcentaje de aportación a la variación total de cada vector fue diferente, es decir, la importancia de las variables cambió de acuerdo con el ambiente de competencia. Dentro del ambiente de libre desarrollo (I), los primeros tres componentes explican $71,9 \%$ del total de la variación observada, en donde el CP1 explica 35,5\% de la variación observada. Los vectores de mayor aportación son: el diámetro a la base, la biomasa de tallo, hojas y ramas y la relación de la cantidad de pigmentos de clorofila (relación de clorofilas $a / b$ ), es decir, variables de crecimiento (Tabla 3), evidenciando el crecimiento inicial como estrategia principal dentro de este ambiente de competencia (I), posteriormente, para el CP2 con 19,1\% del total de la variación, las variables que determinan la arquitectura como: el largo de ramas, la altura de inserción de la rama en el tallo y la biomasa de raíz son las de mayor aportación (Tabla 3); finalmente el CP3, que explica 17,3\% la variable de mayor aportación fue la supervivencia (Tabla 3). Por lo anterior, es posible identificar como estrategia de crecimiento dentro un ambiente de libre desarrollo (ambiente I), el crecer libremente, para posteriormente, cuando los espacios se reducen, los individuos modifican su arquitectura de desarrollo, aumentando la supervivencia de las familias.

Dentro del ambiente de alta densidad inter-familiar (II), los primeros tres componentes explican $77,7 \%$ del total de la variación en dicho ambiente. El CP1 explica
$32,1 \%$ de la variación y los principales vectores de mayor aportación son: altura de inserción de la rama en el tallo, la relación en la cantidad de pigmentos de clorofilas $a / b$, la supervivencia y el largo de rama (Tabla 3). Dentro del CP2 los vectores de mayor aportación fueron la producción de biomasa de hoja, tallo y rama, los cuales explican $27,4 \%$ de la variación observada (Tabla 3). Finalmente, en el CP3 los vectores de mayor aportación son la biomasa de raíz y el diámetro a la base del tallo, con $18,2 \%$ de la variación (Tabla 3). La estrategia de desarrollo de las familias dentro de un ambiente de alta densidad inter-familiar, se basa en la producción de ramas de mayor longitud cerca de la parte apical de la planta, oprimiendo a sus vecinos contiguos. Esto, a su vez, le permite realizar el proceso fotosintético con mayor eficiencia (mayora cantidad de luz solar), aumentando la supervivencia de las familias. Posteriormente, se produce biomasa aérea (tallo, hojas y ramas), aumentando el crecimiento de la planta. Finalmente, aumenta el diámetro del individuo y mejora su sistema de anclaje (sistema radicular).

Para el ambiente de alta densidad intra-familiar (III), los primeros tres componentes explican $72,8 \%$ del total de la variación observada en este ambiente de competencia, en donde el CP1 explica 41,2\% y los vectores que más aportan son: el diámetro a la base del tallo, la biomasa de hojas, tallo y ramas, además de la relación de los pigmentos de clorofila a/b y la altura de inserción de la rama en el tallo (Tabla 3). Para el CP2, los vectores con una aportación mayor fueron: biomasa de raíz y supervivencia, los cuales explican 18,2\% de la variación observada (Tabla 3). Para el CP3, que explica $13,4 \%$, el vector que más aportó fue: largo de la rama (Tabla 3). En este ambiente de competencia, las familias adoptan estrategia de crecimiento; crecer con rapidez, producir ramas cerca de la parte apical del tallo, lo cual aumenta la capacidad fotosintética. Posteriormente, cuando los espacios en la parte aérea se reducen, la producción de un sistema radicular más amplio es esencial, lo cual aumenta la supervivencia de las familias. Finalmente, al aumentar el largo de la rama se busca aumentar el espacio vital de la planta. 
En el presente estudio se determinaron cambios específicos en las familias evaluadas, asociados a un ambiente de competencia, en variables que demuestran el crecimiento, la arquitectura y fisiología básica de los individuos. Las familias presentaron una reducción del crecimiento promedio, menor relación de la cantidad de pigmentos de clorofilas $a / b$ y cambios en la conformación de la arquitectura, finalizando en una menor supervivencia al aumentar la densidad de plantación. Sin embargo, el ambiente de competencia intra-familiar (III), proporcionó un patrón de crecimiento singular, en donde las familias reaccionaron modificando la asignación de biomasa en estructuras como raíz y rama, la relación de la cantidad de pigmentos de clorofilas $a / b$ y el largo promedio de ramas disminuyó. Las variables se agruparon mediante una escala de estrés dentro del ensayo hacia las familias por condición de competencia con cambios en arquitectura y fisiología básica. En conjunto, los resultados demuestran una elevada plasticidad fenotípica de
Pinus pseudostrobus Lindl., cuando se somete a condiciones de alta densidad y a condiciones de competencia intra-familiar.

Los resultados obtenidos determinaron que el ambiente de desarrollo condiciona el éxito adaptativo de ciertos genotipos. También fue posible observar que algunos genotipos tienen la capacidad de modificar parte de su arquitectura y funciones fisiológicas aumentando sus posibilidades de éxito en ambientes adversos. Sin embargo, para enriquecer los resultados obtenidos dentro del presente estudio, es recomendable analizar la respuesta de las familias bajo diferentes ambientes de competencia, tanto de densidad como de tipo de competencia en etapas de campo, y así poder correlacionar ambas etapas, proponiendo la selección temprana de los mejores genotipos (familias) dentro de los diversos ambientes de competencia con el propósito de reducir los tiempos y costos en la evaluación y mantenimiento de los ensayos.

TABla 3. Autovalores y vectores característicos del análisis de Componentes Principales de P. pseudostrobus por ambiente de competencia y aportaciones a la variación total por componente.

\begin{tabular}{|c|c|c|c|c|c|c|c|c|c|}
\hline \multirow{3}{*}{ Variable } & \multicolumn{9}{|c|}{ Ambiente de competencia } \\
\hline & \multicolumn{3}{|c|}{ Ambiente I } & \multicolumn{3}{|c|}{ Ambiente II } & \multicolumn{3}{|c|}{ Ambiente III } \\
\hline & $C P 1$ & $C P 2$ & $C P 3$ & $C P 1$ & $C P 2$ & CP3 & $C P 1$ & $C P 2$ & CP3 \\
\hline Autovalor & 3,1908 & 1,6881 & 1,5420 & 2,8502 & 2,4614 & 1,6220 & 3,7101 & 1,6397 & 1,2093 \\
\hline \multicolumn{10}{|l|}{ Aportación a la varianza } \\
\hline total & $35,5 \%$ & $19,1 \%$ & $17,3 \%$ & $32,1 \%$ & $27,4 \%$ & $18,2 \%$ & $41,2 \%$ & $18,2 \%$ & $13,4 \%$ \\
\hline Diámetro a la base & 0,4217 & 0,2023 & 0,3319 & 0,3041 & 0,2039 & 0,5859 & 0,4262 & 0,0082 & $-0,0304$ \\
\hline Biomasa de raíz & $-0,1245$ & 0,3911 & 0,3611 & $-0,1435$ & $-0,1791$ & 0,6550 & $-0,1344$ & 0,5193 & 0,0766 \\
\hline Biomasa de hoja & 0,4624 & $-0,3037$ & $-0,0161$ & $-0,0371$ & 0,5981 & 0,1369 & 0,3568 & 0,2771 & $-0,5004$ \\
\hline Biomasa de tallo & 0,4664 & $-0,0647$ & 0,1844 & $-0,0772$ & 0,5614 & 0,0994 & 0,4364 & 0,1140 & $-0,2522$ \\
\hline Biomasa de rama & 0,4350 & 0,0770 & $-0,2140$ & $-0,2226$ & 0,4643 & $-0,2485$ & 0,3420 & $-0,3251$ & $-0,2867$ \\
\hline Largo de rama & $-0,0369$ & 0,4611 & $-0,5018$ & 0,3393 & 0,1865 & $-0,1260$ & 0,2207 & 0,3278 & 0,5535 \\
\hline Altura de inserción de rama & 0,1010 & 0,6609 & $-0,0727$ & 0,5367 & $-0,0056$ & 0,1508 & 0,3210 & 0,2887 & 0,2323 \\
\hline Relación de clorofilas $a / b$ & $-0,3828$ & $-0,1909$ & $-0,0427$ & 0,4398 & $-0,0102$ & $-0,3079$ & 0,4250 & $-0,0720$ & 0,3641 \\
\hline Supervivencia & $-0,1665$ & 0,1333 & 0,6483 & 0,4829 & 0,0526 & $-0,0609$ & 0,1815 & $-0,5819$ & 0,3223 \\
\hline
\end{tabular}

NOTA: CP 1= Componente principal 1, CP 2= Componente principal 2 y CP 3=Componente principal 3;

NOTA: Mayores aportaciones dentro del componente principal se resaltan en negritas. 


\section{CONCLUSIONES}

La reducción del espacio fue lo que más influyó entre tratamientos en las variables evaluadas.

El crecimiento y el ajuste fisiológico en la relación de la cantidad de pigmentos de clorofilas $a / b$, fueron la evidencia más clara en la agrupación de los ambientes de competencia.

Se observaron distintos patrones en el desarrollo de las familias dentro de los diversos ambientes de desarrollo, con los cambios más drásticos dentro de un ambiente de alta densidad intra-familiar, representando un ambiente de mayor estrés para las familias evaluadas.

Se observó un elevado nivel de plasticidad fenotípica en las familias evaluadas, expresado en cambios en la arquitectura, supervivencia y ajuste fisiológico.

\section{RECONOCIMIENTOS}

Este proyecto fue financiado con fondos del proyecto de Fondos Mixtos Conacyt-Gobierno del Estado de Michoacán clave 63532 y con beca-Conacyt para el primer autor. Escritura y correcciones con proyecto de estancia posdoctoral Conacyt para el primer autor.

\section{REFERENCIAS}

Adams, W.T., J.H. Roberts y B.J. Zobel. 1973. Intergenotypic interactions among families of Loblolly Pine (Pinus taeda L.). Theoretical and Applied Genetics 43:319-322.

Barthélémy, D., F.Blaise, T. Fourcaud, y E. Nicolini. 1995. Modelisation et simulation de l'architecture des arbres: Bilan et perspectives, Revue Forestière Française XLVII:71-96.

Cambrón-Sandoval, V.H., M.L. España-Boquera, N. SánchezVargas, C. Sáenz-Romero, J.J. Vargas-Hernández e Y. Herrerías-Diego. 2011. Producción de clorofila en Pinus pseudostrobus en etapas juveniles bajo diferentes ambientes de desarrollo. Revista Chapingo Serie Ciencias Forestales y del Ambiente 17(2):253-260.

Cambrón-Sandoval, V.H., N. Sánchez-Vargas, C. SáenzRomero, J.J. Vargas-Hernández, M.L. España-Boquera e Y. Herrerías-Diego. 2013a. Genetic growth parameters in Pinus pseudostrobus families under different competitive environments. New Forests 44:219-232.
Cambrón-Sandoval, V.H., H. Suzán-Azpiri, J.J. VargasHernández, N. Sánchez-Vargas y C. Sáenz-Romero. 2013 b. Strategies for growth and distribution of biomass in Pinus pseudostrobus under different conditions of competition. Revista Fitotecnia Mexicana 36 (1):71-79.

Ferreira, M., H.T.Z. Couto y J. Mascarenhas S. 1972. The introduction of Mexican pines into the region of Poços de Caldas. IPEF Piracicaba 4:95-109.

Godin, C., 2000. Representing and encoding plant architecture: A review. Annals of Forest Science 57:413-438.

Harms, R.W., D.C. Whitesell y S.D. DeBell. 2000. Growth and development of loblolly pine in a spacing trial planted in Hawaii. Forest Ecology and Management 126:13-24.

Harms, W.R., D.S. DeBell y C.D. Whitesell. 1994. Stand and tree characteristics and stockability in Pinus taeda plantations in Hawaii and South Carolina. Canadian Journal of Forest Research 24:511-521.

Jackson, D.A. 1993. Stopping rules in principal components analysis: a comparison of heuristical and statistical approaches. Ecology 74:2204-2214.

Jeffers, J.N., 1967. Two Case Studies in the Application of Principal Component Analysis. Journal of the Royal Statistical Society. Series C (Applied Statistics) 16(3):225-236.

Jolliffe, I.T. 1986. Principal component analysis. Springer-Verlag. Nueva York, 271 p.

Kaiser, H.F. 1958. The Varimax criterion for analytic rotation in factor analysis. Psychometrika 23:187-200.

Kuuluvainen, T., 1992. Tree architectures adapted to efficient light utilization: is there a basis for latitudinal gradients?. Oikos 65:275-284.

Liu, C.J. y T.D. Keister. 1978. Southern pine stem form defined through principal component analysis. Canadian Journal of Forest Research 8:188-197.

Mutke, S., R. Sievänen, E. Nikinmaa, J. Perttunen y L. Gil. 2005. Crown architecture of grafted Stone pine (Pinus pinea L.): shoot growth and bud differentiation. Trees Structure and Function 19(1):15-25.

Nishimura, T.B. y E. Suzuki. 2001. Allometric differentiation among tropical tree seedlings in heath and peatswamp forests. Journal of Tropical Ecology 17:667-681. 
Noland, T.L., G.H. Mohammed y R.G. Wagner. 2001. Morphological characteristics associated with tolerance to competition from herbaceous vegetation for seedlings of jack pine, black spruce and white pine. New Forests 21:199-215.

Perry, J.P., 1991. The pines of Mexico and Central America. Timber Press. Portland, Oregon, EUA. 231.

Pires, C., 1987. Complexo de Pinus pseudostrobus em Campos do Jordão e Itararé. In: Simposio sobre silvicultura y mejoramiento genético de especies forestales. Centro de Investigaciones y Experiencias Forestales 5:150-61.

Pla, L. 1986. Análisis Multivariado: Método de Componentes Principales. Instituto Interamericano de Estadística. Sec. General de la OEA Washington, D.C.89 p.

Real, P.L., J. A. Moore y J.D. Newberry. 1989. Principal components analysis of tree stem profiles. Canadian Journal of Forest Research 19:1538-1542.

Rodríguez-García, E., F. Bravo y T. Spies. 2011. Effects of overstorey canopy, plant-plant interactions and soil properties on Mediterranean maritime pine seedling dynamics. Forest Ecology and Management 262:244-251.

Sánchez-Gómez, D., T. Velasco-Conde, F.J. Cano-Martín, M.A. Guevara, M.T. Cervera e I. Aranda. 2011. Interclonal variation in functional traits in response to drought for a genetically homogeneous Mediterranean conifer. Environmental and Experimental Botany 70:104-109.
Sánchez-Vargas, N.M. y J.J Vargas-Hernández. 2007. Competencia y su relación con los parámetros genéticos en clones de eucalipto. Ciencia e Investigación Forestal-Instituto Forestal/Chile 13(2):361-369.

SAS Institute, INC. 1999. SAS / STAT® User's Guide For Personal Computers, Version 8, Cary. Carolina del Norte, EUA 3884 p.

Tardif, J., J.J. Camarero, M. Ribas y E. Gutiérrez. 2003. Spatiotemporal variability in tree growth in the central Pyrenees: climatic and site influences. Ecological Monographs $73: 2,241-257$.

Wright, I.J. y M. Westoby. 2001. Understanding seedling growth relationships through specific leaf area and leaf nitrogen concentration: generalisations across growth forms and growth irradiance. Oecologia 127:21-29.

Wrigth, J. A. y A. Wessels. 1992. Laboratory scale pulping of Pinus pseudostrobus, P. maximinoi and P. patula. Instituto de Pesquisas Florestais (IPEF International), Piracicaba 2:39-44.

Manuscrito recibido el 29 de octubre de 2012. Aceptado el 16 de agosto de 2013.

Este documento se debe citar como:

Cambrón-Sandoval, V.H., H. Suzán-Azpiri, C. Sáenz-Romero y N.M. Sánchez-Vargas. 2014. Desarrollo de Pinus pseudostrobus bajo distintos ambientes de crecimiento en jardín común. Madera y Bosques 20(1):47-57. 